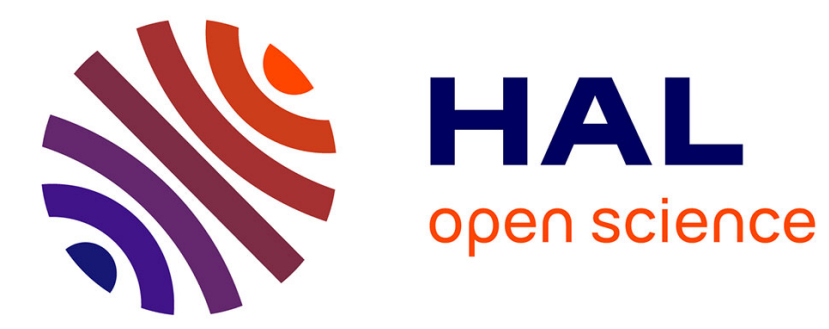

\title{
Les potins d'Alésia
}

Brigitte Fischer

\section{To cite this version:}

Brigitte Fischer. Les potins d'Alésia. Gallia - Archéologie de la France antique, 1995, Les potins gaulois, 52, pp.37-42. 10.3406/galia.1995.3120 . hal-01911257

\section{HAL Id: hal-01911257 \\ https://hal.science/hal-01911257}

Submitted on 7 Feb 2020

HAL is a multi-disciplinary open access archive for the deposit and dissemination of scientific research documents, whether they are published or not. The documents may come from teaching and research institutions in France or abroad, or from public or private research centers.
L'archive ouverte pluridisciplinaire HAL, est destinée au dépôt et à la diffusion de documents scientifiques de niveau recherche, publiés ou non, émanant des établissements d'enseignement et de recherche français ou étrangers, des laboratoires publics ou privés.

\section{(a)(1) $\$$}

Distributed under a Creative Commons Attribution - NonCommercial - NoDerivatives $\mid 4.0$ 


\section{LES POTINS, TRACEURS CHRONOLOGIQUES?}

La chronologie absolue des potins, comme celle de l'ensemble du mobilier de La Tène finale, est encore appelée à évoluer, mais nous avons ici établi les conditions d'une éventuelle chronologie relative en distinguant des classes à l'intérieur de certaines séries de potins : potins « à la grosse tête », potins « à la tête diabolique ", potins " au personnage courant ", potins " au sanglier » (Scheers, 1983, p. 712-729, S 186), potins au "rameau » (Scheers, 1983, p. 735-748).

Une fois défini le classement typologique d'une série de potins, l'association des différents types à des horizons stratigraphiques bien définis devrait permettre de redonner à ce mobilier archéologique sa place de traceur chronologique. Certes, l'état de conservation de ces objets les rend parfois difficilement identifiables et ils sont moins communément présents en stratigraphie que la céramique. En revanche, comme tous les objets monétiformes fabriqués en série, ce sont des séries reproductibles pour lesquelles il est toujours possible d'aboutir à une proposition de chronologie relative dès que le matériel étudié est statistiquement représentatif. On a donc beaucoup à attendre de l'analyse des faciès monétaires des sites et encore plus des niveaux stratigraphiques lorsque le nombre de monnaies le permet (Colbert de Beaulieu, 1973).

\subsection{Les potins d'Alésia}

\section{Brigitte Fischer}

Résumé. 1805 pièces gauloises ont été recueillies à Alise-Sainte-Reine et dans les environs. Elles se répartissen en trois grands lots : le musée d'Alise, le musée des Antiquités nationales à Saint-Germain-en-Laye et le numéraire provenani des fouilles de J. Bénard. Cette récolte se décompose ainsi : 3 monnaies d'or ou d'électrum, 376 pièces d'argent, 265 bronzes frappés, 190 bronzes ou potins (leur état ne permet pas de trancher) et 971 potins. Le premier lot, celui du musée d'Alise, comporte 802 potins, le deuxième (musée des Antiquités nationales) 62, le troisième (fouilles Bénard) 107. Le numéraire lingon est largement dominant au musée d'Alise et dans les fouilles Bénard, alors que les Sénons sont les plus représentés au musée des Antiquités nationales. Le nombre élevé des potins attribués aux Séquanes pose un problème intéressant. Les pièces conservées au musée d'Alise proviennent presque toutes de la ville gallo-romaine, nous ne possédons pas de renseignements stratigraphiques pour les espèces qui constituent la collection du musée des Antiquités nationales, mais leur perte est normalement datable de 52 avant J.-C. Quelques monnaies des fouilles Bénard ont été recueillies dans un horizon stratigraphique daté entre 70 et 40, les autres sont issues d'un contexte gallo-romain.

Zusammenfassung. 1805 keltische Münzen sind in Alise-Sainte-Reine und Umgebung gefunden worden. Sie verteilen sich auf drei große Bestände: Museum Alise, musée des Antiquités nationales in St. Germain-en-Laye und die Stücke aus den Grabungen J. Bénard. Es handelt sich um 3 Münzen aus Gold oder Elektrum, 376 aus Silber, 265 Bronzeprägungen, 190 Münzen aus Bronze oder Potin (ihre Erhaltung erlaubt keine klare Zuweisung) und 971 Potinmünzen. Die Sammlung in Alise enthält 802 Potinmünzen, das musée des Antiquités nationales 62 und aus den Crabungen Bénard stammen 107 Potinmünzen. Münzen, die den Lingonen zugewiesen werden, dominieren im Museum von Alise sowie den Cirabungen Bénard, dagegen die sog. Senonen-Münzen im musée des Antiquités nationales. Die hohe Zahl von Sequaner-Münzen verdient Interesse. Die Stücke im Museum von Alise stammen fast alle aus dem Bereich der gallorömischen Siedlung, für jene des musée des Antiquités nationales besitzen wir keine stratigraphischen Angaben, doch dürften sie allgemein im Jahr 52 v. Chr. in den Boden gelangt sein. Einige Münzen der Crabungen Bénard stammen aus einem zwischen 70 und 40 v. Chr. datierbaren Horizont, die übrigen aus einem gallo-römischen Kontext. 
Mille huit cent cinq monnaies gauloises ont été recueillies à Alise-Sainte-Reine et dans les environs. Elles se répartissent actuellement en trois grands lots : le musée d'Alise, qui comporte mille cent quatre-vingt-cinq pièces, le musée des Antiquités nationales à Saint-Germain-enLaye : quatre cent soixante-deux monnaies et le numéraire provenant des fouilles de Jacky Bénard au Centre public : cent cinquante-huit pièces, qui entreront prochainement au musée d'Alise. Cette récolte se décompose ainsi : trois monnaies d'or ou d'électrum, trois cent soixante-seize exemplaires en argent, deux cent soixantecinq bronzes frappés, cent quatre-vingt-dix bronzes ou potins (leur état ne permettant pas de trancher) et neuf cent soixante et onze potins, dont cent trente-huit exemplaires non identifiables.

Les figures 12 et 13 présentent les types des potins trouvés à d'Alésia.

Les deux lots les plus importants, ceux du musée d'Alise et des fouilles de J. Bénard, sont essentiellement issus d'un horizon gallo-romain; en revanche, on admet que les espèces conservées au musée des Antiquités nationales proviennent des fossés du siège et ont été perdues en 52 avant J.-C. Cette collection, qui est la plus réduite et la plus ancienne, comporte quatre cent soixante-deux pièces. Trois sont en or ou électrum, deux cent quarantetrois en argent, cent cinquante-quatre en bronze et soixante-deux sont des potins. Il y a une représentation dominante des Sénons, puisque leur numéraire constitue exactement la moitié de l'ensemble : trente et une pièces étant de type LT XXX, 7434. Viennent ensuite les Iingons avec treize monnaies, quatre sont de type LT XXXIII, 8319 et neuf de type ITT XXXIII, 8329, soit environ $20 \%$ du total. Pour le reste, il y a une grande dispersion des attributions. On trouve trois potins « à la tête diabolique " des Turons (type BN 5677-5690) ${ }^{19}$ ex aequo avec les potins de "type Mâlain " ${ }^{20}$. I.es autres numéraires sont représentés par une ou deux pièces. Notons au passage qu'il y a une monnaie "à la grosse tête " de type LT XVI, 5368 et deux exemplaires qui sont de " type Alésia ». Cinq potins n'ont pu être identifiés en raison de leur état.

19. Muret, Chabouillet, 1889 , p. 127.

20. C'est sur le site de Mâlain (Côte-d'Or) que nous avons rencontré pour la première fois ce numéraire. Cette appellation est donc arbitraire et elle sera remplacée par l'attribution à un peuple, dès qu'il sera possible de l'établir. Les quelques exemplaires connus ont été le plus souvent donnés aux Séquanes.
$\mathrm{Si}$ nous considérons maintenant le gros ensemble constitué par les monnaies du musée d'Alise et le numéraire des fouilles de Jacky Bénard, nous avons un total de neuf cent neuf potins qui se répartissent ainsi : quatre cent soixante-dix exemplaires lingons (242 pièces de type LT 8319 et 228 de type LT 8329) soit plus de $50 \%$ du total. I.es monnaies attribuées aux Séquanes arrivent en deuxième position avec cent quatre-vingts exemplaires. À vrai dire, des distinctions importantes doivent être faites à l'intérieur de ce monnayage. On trouve dix-huit pièces de type LT 5368 " à la grosse tête " et quatre-vingt-six monnaies très proches des précédentes, mais qui présentent des différences constantes et que nous proposons d'appeler provisoirement "type Alésia " ${ }^{21}$. I a tête est plus grosse que dans la série LT 5368, elle occupe tout le champ et le quadrupède, au lieu d'être dressé sur ses pattes avant, a le corps à peu près parallèle à la ligne de sol; sa queue est bouletée. À ces quatre-vingt-six exemplaires, il faut ajouter une partie des quarante pièces qui ne peuvent être déterminées avec certitude et sont soit des potins de type LT 5368, soit des potins de " type Alésia ». Il paraît donc raisonnable de considérer que ces derniers représentent une centaine d'exemplaires. Jusqu'à présent, aucun site ne semble en avoir livré autant. Par ailleurs, un rebut de fabrication conservé au musée, semble bien appartenir à ce monnayage qu'il est tentant de considérer comme une production locale.

Les autres potins séquanes sont, pour la plupart, dérivés du type dit «à la grosse tête ». Les deux séries les plus abondantes correspondent à LT XVI, 5393 : onze exemplaires et LT XVI, 5401 : dix exemplaires. On trouve encore six pièces de type LT XVI, 5390, deux de type LT XVI, 5527 et une de chacun des types suivants : LT XVI, 5542, LT XVI, 5611 et 5629. Deux potins, enfin, n'ont pas pu être identifiés. Le numéraire sénon totalise soixante-dix-sept pièces. Comme au musée des Antiquités nationales, le type le plus fréquent correspond à LT 7434. On compte soixante-dix monnaies de cette catégorie. Les autres pièces sont un potin de type I.T XXX, 7417, trois de type LT XXX, 7437, deux de type IT XXX, 7445 et un non identifié.

Les Éduens et les Leuques sont à égalité avec quinze exemplaires. Pour les Éduens, on trouve dix potins de

21. Alésia est à notre connaissance le lieu qui a livré le plus grand nombre de pièces de cette catégorie. Elles correspondent au type A9, de A. Gciscr ct K. Grucl. 

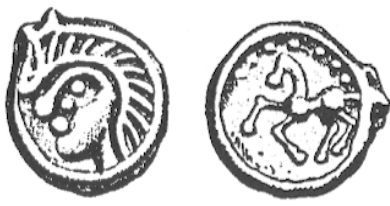

LT 7434. Sénons

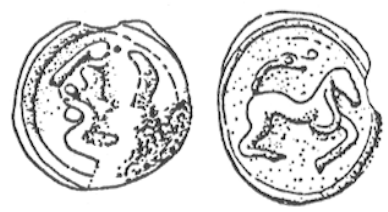

Type Mâlain

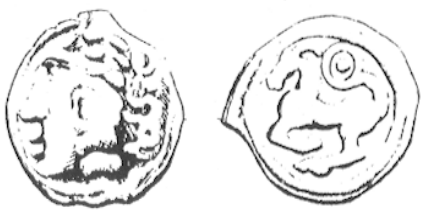

LT 5393. Séquanes

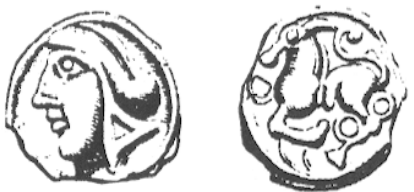

LT 5527. Séquanes
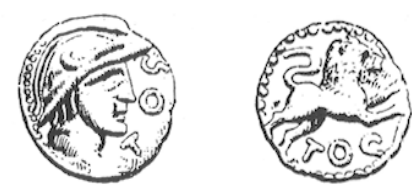

LT 5629. Séquanes

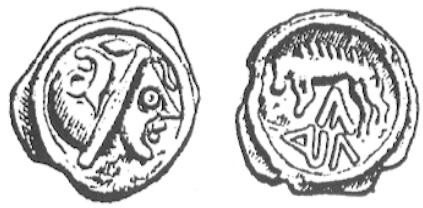

LT 8319. Lingons

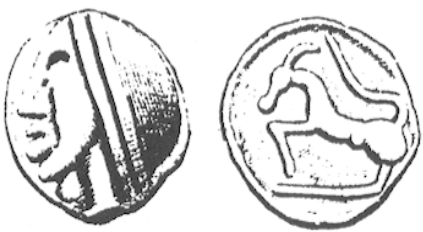

LT 5368. Séquanes

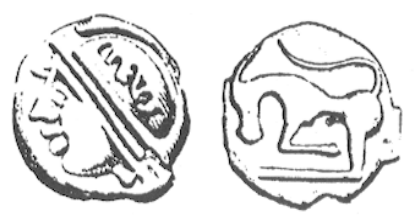

LT 5401. Séquanes

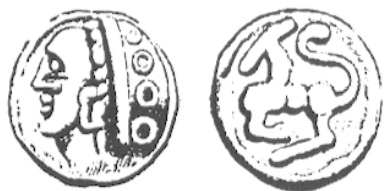

LT 5542. Séquanes
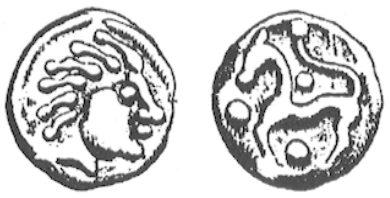

LT 7417. Sénons

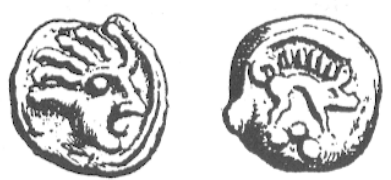

LT 7445. Sénons

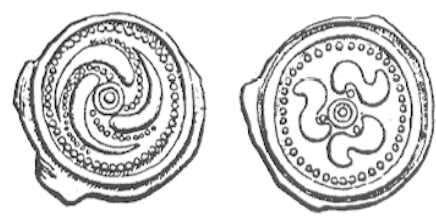

LT 8329. Lingons

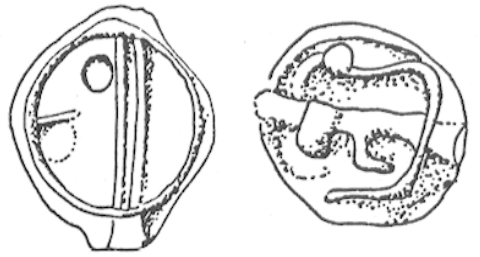

Type Alésia

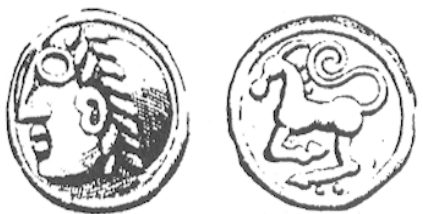

LT 5390. Séquanes

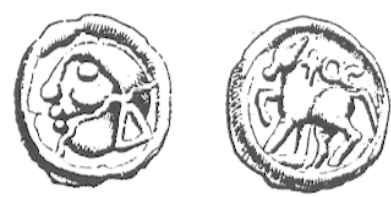

LT 5611. Séquanes

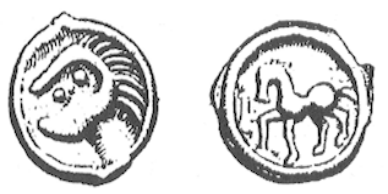

LT 7437. Sénons

Fig. 12. Types monétaires présents à Alésia. 


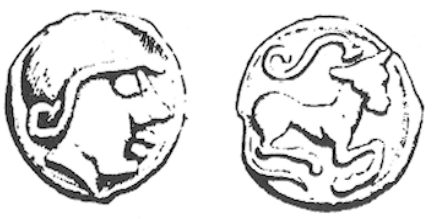

LT 5253. Eduens

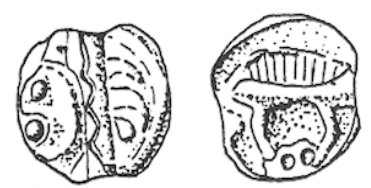

Type du Tremblois
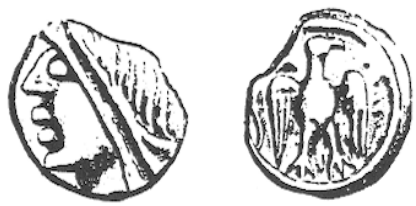

LT 5275. Carnutes/Eduens

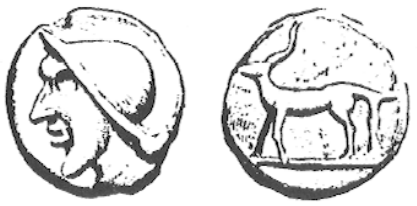

LT 5267. Eduens

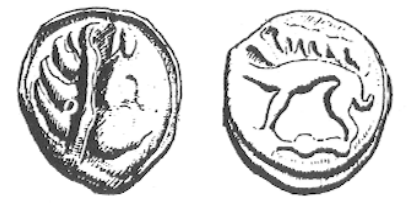

LT 9147. Leuques
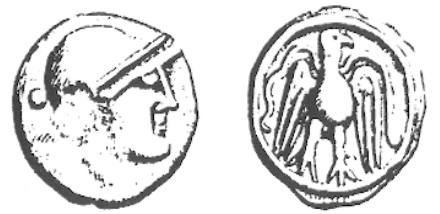

LT 5277. Carnutes/Eduens

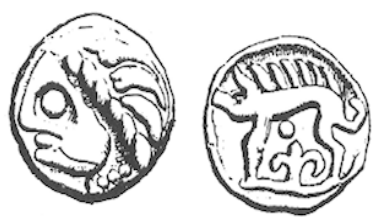

LT 9078. Leuques

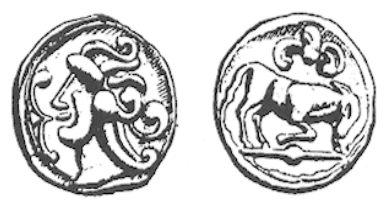

LT 9155. Leuques
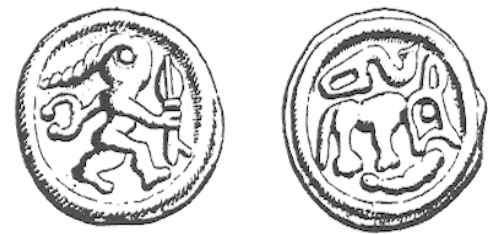

LT 8124. Rèmes

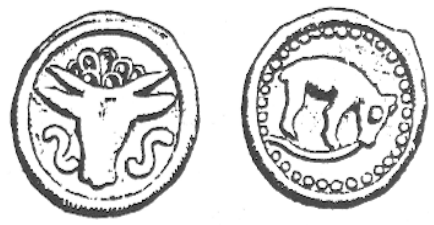

LT 8351. Rèmes

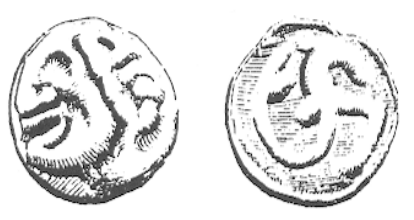

LT 2935. Allobroges/Eduens

Fig.13. Types monélaires présents à Alésia (suite).

type LT XVI, 5253, un exemplaire de type XVI, 5267, deux monnaies au cou triangulaire et an quadrupède, ainsi qu'un potin non identifié ${ }^{22}$. Le numéraire leuque n'apparaît absolument pas dans les fouilles de Jacky Bénard. Il est présent au musée, où l'on recense douze monnaies de type LT XXXVII, 9078, onze d'entre elles présentant un aspect un peu particulier : le diadème est fait d'un zigzag en creux, au lieu d'être constitué d'une sorte de torsade. Les pièces de ce type sont nombreuses au sanctuaire du Tremblois, en forêt de Châtillon, on

22. Des monnaies de ce type ont été trouvées au Grand-Saint-Bernard et à La Tène : nous remercions A. Geiser de cette information. peut donc s'interroger sur le lieu exact de fabrication de cette série, dont on trouve un certain nombre d'exemplaires en territoire lingon, assez loin de la cité des Leuques. Il existe, par ailleurs, au musée d'Alise-SainteReine, un potin de type IT XXXVII, 9147 et deux potins qui sont des variantes du type LT XXXVII, 9155.

Les autres monnayages sont représentés par quelques pièces (de une à trois). C'est ainsi qu'il y a deux potins « à la tête diabolique » des Turons, trois potins de type LT XVI, 5275, qui appartiennent aux Carnutes ou aux Éduens, un potin de type LT XVI, 5277, qui présente la même incertitude d'attribution. Les Rèmes ont fourni trois monnaics : dcux dc type LT XXXII, 8124 et une au 
bucrane de type LT XXXIII, 8351. On trouve aussi trois petits potins bituriges : un exemplaire " au swastika " (type BN 6284-6294) ${ }^{23}$ et deux " au quadrupède " (type BN 5653) ${ }^{24}$, ainsi qu'un potin de " type Mâlain ". Pour clôturer la série, nous avons un potin de type IT VII, 2935 " à l'hippocampe ", attribué aux Allobroges, mais qui pourrait bien être éduen, un potin qui serait une imitation de monnaie « à la croix » et une pièce inédite.

Après l'étude typologique, les informations chronologiques doivent être examinées. Les monnaies du musée de Saint-Germain-en-Laye ont été recueillies au cours des fouilles de Napoléon III, de 1862 à 1865 . L'empereur fit dégager les fossés de la ligne de circonvallation et d'autres travaux de siège des Romains. C'est au pied du mont Réa, dans le fond du fossé inférieur limitant le camp D du côté de la rivière, que l'on a découvert six cent huit pièces gauloises et romaines. L'authenticité de cette récolte a parfois été mise en doute. Il semble bien établi maintenant que c'est à tort. Les monnaies romaines apportent une confirmation de poids à la datation, puisque la plus récente est de 54 avant J.-C. ${ }^{25}$. Les pièces recueillies en ce lieu appartenaient, selon toute vraisemblance, aux hommes qui constituaient l'armée de secours.

Le lot de J. Bénard (158 monnaies, dont 107 potins), provient du Centre public d'Alise ${ }^{26}$. Dans ses fouilles, J. Bénard distingue trois horizons archéologiques, qu'il a appelés " occupations 1,2,3 ", la première occupation étant subdivisée en deux parties : la et 1b. L'occupation 1 dans sa totalité est datée entre $70 / 60$ et $40 / 30$ avant J.-C., sans qu'il soit possible de différencier la et $1 \mathrm{~b}$ sur le terrain. À vrai dire, c'est essentiellement sur la base de la numismatique que la distinction a été faite : «La première phase (Oc. 1a) se place dans la première moitié du $\mathrm{I}^{\mathrm{er}}$ s. avant J.-C., sans doute peu avant la guerre des Gaules du fait de la présence de potins; la seconde phase (Oc. 1$1 \mathrm{~b}$ à $2 \mathrm{c}$ ) dans la seconde moitié de ce siècle, la transition $1 \mathrm{a}-1 \mathrm{~b}$ correspondant sans doute à la guerre des Gaules ${ }^{27}$. Les monnaies recueillies dans l'Oc. la sont quatre deniers éduens, trois deniers lingons, un denier non identifié, un

23. Muret, Chabouillet, 1889 , p. 139.

24. Muret, Chabouillet, 1889, p. 126.

25. S. SCHEERS, La numismatique d'Alésia : quelques précisions, in : Mélanges offerts au docteur J.-B. Colbert de Beaulieu, Paris, 1987, p. 743-752.

26. Nous remercions sincèrement $\mathrm{J}$. Bénard de nous avoir confié ses monnaies pour étude.

27. J. BÉNARD, Les niveaux précoces du centre public d'Alésia, thèse de $3^{\mathrm{e}}$ cycle, université de Dijon, 1989, p. 135 (dactyl.). bronze arverne, deux potins séquanes (l'un est de " type Alésia ", l'autre est de type LT 5401), ainsi que quatre potins non identifiés. Le mobilier qui caractérise la totalité de cette occupation 1 est constitué par un monnayage exclusivement gaulois, les potins représentent environ $50 \%$ du lot, les deniers $35 \%$, le reste est constitué de bronzes frappés. Les deux tiers des fibules sont en fer. Les cinq fibules identifiées sont de type filiforme (2 ou 3), Nauheim ( 1 ou 2) et Feugère 3a (1). La céramique fine d'importation est exclusivement de la céramique à vernis noir, campanienne $A$, mais surtout $B$ de type Calès. Les formes sont, pour la moitié, des Lamboglia 5, mais ce qui est caractéristique, c'est la forte proportion de Lamboglia $1(25 \%)$.

La céramique commune est composée à plus de $80 \%$ de carbonifère; la céramique " lie de vin " représente plus de $5 \%$ des tessons; la céramique à pâte fine homogène et parois noires lustrées ne se trouve que dans cette occupation; environ $90 \%$ des vases sont des formes basses et plus de la moitié des fonds sont plats.

Dans la phase $1 \mathrm{~b}$, la proportion de potins s'accroît considérablement et atteint $75 \%$. Le fait nouveau est l'apparition de monnaies gallo-romaines à légende GERMANVS INDVTILLI I ${ }^{28}$, de demi-dupondii de Nîmes et du monnayage augustéen.

Les monnaies du musée d'Alise-Sainte-Reine constituent le lot le plus important, cent vingt-quatre pièces ont été recueillies dans les fouilles du quartier artisanal, effectuées par Michel Mangin. I.es autres sont pratiquement toutes dépourvues de provenances précises, mais Élisabeth Rabeisen, conservateur du musée, considère qu'elles proviennent en quasi-totalité de la ville gallo-romaine ${ }^{29}$.

Dans le quartier de commerçants et d'artisans, $M$. Mangin a distingué six horizons archéologiques. L'horizon I a été divisé en Ia et Ib. L'auteur indique que dans l'horizon Ia des vestiges structurels et mobiliers exclusivement indigènes sont regroupés, en notant qu'historiquement il doit être surtout situé au début de l'occupation romaine. Il ajoute : "Il n'existe pas dans le quartier une occupation attestée de l'indépendance séparée de l'occupation de la seconde moitié du $\mathrm{I}^{\mathrm{er}} \mathrm{s}$. av. J-C. ».

" L’horizon Ib présente des éléments de deux types intimement mêlés ou seulement juxtaposés : des vestiges

28. I a création de ce numéraire est datéc aux environs de 10 avant J.-C. 29. Nous exprimons notre reconnaissance à É. Rabeisen, qui nous a permis d'étudier les monnaies du musée et nous a fourni cette information. 
identiques ou analogues à ceux de l'horizon précédent et d'autres manifestant un apport nouveau et extérieur; c'est un horizon mixte, encore indigène par beaucoup de traits, mais déjà gallo-romain par d'autres. Ces deux premiers horizons ont èté regroupés sous le même chiffre parce qu'ils sont en continuité l'un avec l'autre et nettement séparés des horizons ultérieurs de structure exclusivement gallo-romaine. ${ }^{30}$

Cette première occupation paraît donc contemporaine de la guerre des Gaules. Dans l'horizon Ia, on a recueilli soixante-trois monnaies gauloises (sur un total de cent vingt-quatre), soit la moitié.

Dans l'horizon Ib, on a trouvé vingt-six pièces, ce qui est moins de la moitié de la récolte précédente, mais autant que pour les horizons postérieurs réunis.

La première occupation regroupe donc les trois quarts des monnaies gauloises. L'horizon Ia a fourni seize monnaies des Lingons : un denier de Kaletedou et quinze potins (douze sont de type LT 8319 et 3 de type 8329 ). Les Séquanes sont presque à égalité avec quinze pièces : un denier d'argent et quatorze potins ainsi répartis : douze du type "à la grosse tête ", IT 5368 (ou «type Alésia »), un de type LT 5390 et un de type 5401. Les autres peuples sont représentés par une ou deux monnaies : deux deniers éduens, un denier et un potin des Leuques, un potin qui semble attribuable aux Suessions et trente-sept pièces non identifiées.

Dans l'horizon 1b, les Séquanes disparaissent et les Lingons sont encore majoritaires. Sur vingt-six pièces, onze appartiennent aux Lingons. Il y a un denier et dix potins (deux exemplaires LT 8319 et huit exemplaires LT 8329). On trouve, par ailleurs, un bronze biturige, un denier d'argent non attribué à légende ARIVOS / SANTONOS, un bronze carnute et un potin turon (probablement « à la tête diabolique ») ; sept monnaies, enfin, restent sans identification.

À partir de l'ensemble de ces éléments, il est possible dc tirer quelques conclusions qui donnent une meilleure connaissance des potins :

1 , au point de vue chronologique : l'horizon le plus ancien de découverte des bronzes coulés est l'occupation la de J. Bénard, au Centre public. Nous avons vu, d'une part, que son début était daté de $70 / 60$, ce qui n'est vraiment pas éloigné de la guerre des Gaules, d'autre part que c'était essentiellement la présence de potins qui faisait placer cet horizon avant la guerre plutôt que pendant la guerre;

2 , au point de vue typologique : il semble que les monnaies d'Alise-Sainte-Reine apportent des pistes de recherche intéressantes pour trois catégories de pièces :

a, les potins du «type Mâlain " représentés à quelques exemplaires dans la région et aussi dans le Loiret. Ils étaient jusqu'à présent, le plus souvent classés avec les potins séquanes, dont ils sont, en effet très proches, ou avec les potins « à la tête diabolique " des Turons.

$b$, les potins attribués aux Leuques, qui diffèrent de la série classique LT 9078, en particulier à cause du diadème qui orne la tête. Il ne faut pas oublier qu'il y en a ici onze exemplaires sur dix-sept et qu'ils sont abondants sur le site voisin du Tremblois. Cette série mérite donc une recherche plus poussée.

c, le numéraire le plus intéressant, enfin, est constitué par les potins de "type Alésia ", dérivés du type « à la grosse tête ». Ils représentent plus de la moitié des monnaies attribuées aux Séquanes et sont présents dans les trois lots. Deux exemplaires figurent dans la collection du musée des Antiquités nationales. Il semble donc qu'en -52 ils viennent d'apparaître et on constate leur montée en force au début de l'époque gallo-romaine. En attendant des investigations plus poussées, il paraît possible de les considérer comme un monnayage mandubien. Il a été utilisé, à l'origine, parallèlement au numéraire lingon et il sera peu à peu supplanté par lui. Dans les niveaux la de $\mathrm{J}$. Bénard et de M. Mangin, Lingons et Séquanes sont à peu près à égalité. Dans le niveau $1 \mathrm{~b}$ de J. Bénard, les monnaies "séquanes" représentent la moitié des monnaies lingones; dans le niveau $1 \mathrm{~b}$ de M. Mangin, le numéraire "séquane " disparaît : voilà des informations fort utiles qui permettent de mieux cerner l'émission de ce numéraire, en attendant que son étude approfondie soit effectuée. 